\title{
Tabletop Prototyping of Serious Games for 'Soft Skills' Training
}

\author{
Conor Linehan, Shaun Lawson and Mark Doughty \\ Department of Computing and Informatics \\ University of Lincoln \\ Lincoln, UK \\ clinehan@lincoln.ac.uk
}

\begin{abstract}
Serious games offer a relatively low cost, highly engaging alternative to traditional forms of soft skills training. The current paper describes an approach taken to designing a serious game for the training of soft skills. A tabletop prototype of the game was created and evaluated with a group of 24 participants. Initial findings suggest that the game successfully created an environment in which it was advantageous to engage in appropriate collaborative decision making behaviors, as well as providing built-in opportunities for a tutor to guide under-performing groups.
\end{abstract}

Key Words - Game design, serious games methodologies, education and learning, collaborative games, soft skills.

\section{INTRODUCTION}

The management of events, people or organizations in dynamically changing environments requires both knowledge of pre-defined plans and procedures, and 'softer' skills such as; communication and understanding of information under conditions of stress, problem-solving with partial or contradictory information, and decisionmaking in the face of competing demands [1]. Programs designed to train soft skills should ideally expose students to exactly those sorts of stressful, uncertain and dynamically changing environments [2]. Constructing such training exercises with traditional role-playing methodologies can be an expensive, laborious task, which furthermore lacks the engagement inherent in a real scenario [3]. However, modern computer game technology appears to represent an ideal tool for presenting and controlling complex, engaging and dynamically changing environments and may prove to be useful tools in the training of soft skills $[3,4]$.

The work described here is being conducted as part of an EU LEONARDO project named DREAD-ED, which aims to provide game-based soft skills training to emergency management personnel (see http://www.dreaded.eu for further information). Emergencies generally constitute unpredictable, stressful environments and are managed by groups of people drawn from various disciplines and agencies. Importantly, the process of managing an emergency requires group members to communicate effectively and engage in collaborative decision making in order to solve the problems presented [5].

This paper presents the process of designing computer game to function as part of a soft skills training program, as well as an evaluation of a table top game designed to engage players in appropriate collaborative decision making behavior. Some brief conclusions and an outline of proposed future work are also presented.

\section{BACKGROUND}

While the term 'soft skills' is not common within social psychology literature, a large amount of research has been carried out on precisely these skills, and many factors have been judged to play a role in the quality of collaborative decisions made by groups. The literature suggests that "group decision making can be biased and ineffective and that conditions must be created for group members to share information and make decisions [6]." It is suggested that efforts must be made to specifically train group members in the process of effective group decisionmaking. For example, groups that are characterized by an equality of member participation make higher quality decisions than groups that are dominated a minority of individuals [7]. Thus, groups should be trained to adopt a systematic structure for discussions that promote participation and sharing of information by all group members [7].

Little research has been conducted on designing games specifically as tools for training soft skills. However, research has indicated that computer games can prove to be innovative and powerful tools for learning and education, if appropriate attention is given to incorporating "sound cognitive, learning, and pedagogical principles into their design and structure [8]." Unfortunately, a large number of educational, or serious, games appear to have ignored the principles that typically inform educational materials [8], particularly in regard to two issues; 1) embedding learning outcomes within the game mechanics [9], and, 2) providing immediate and specific feedback to participants regarding their behaviour [10].

Bogost [9] describes the process of embedding the learning outcomes of a serious game within the game play mechanics as constituting procedural rhetoric. The author describes a number of serious games that are deficient in procedural rhetoric (p. 49-51). These games simply borrow the game play mechanics of traditional games and apply novel graphical skins to them. It is entirely possible to play these games successfully while remaining oblivious to the desired learning outcomes. The learning is not embedded within the game play mechanics, rather the player is presumed to infer the correct message from playing the game. Conversely, Bogost mentions a number of games, such as The McDonalds Videogame (p. 29) which excels in embedding the learning outcomes within the game mechanics, thus constituting superior tools for education. 


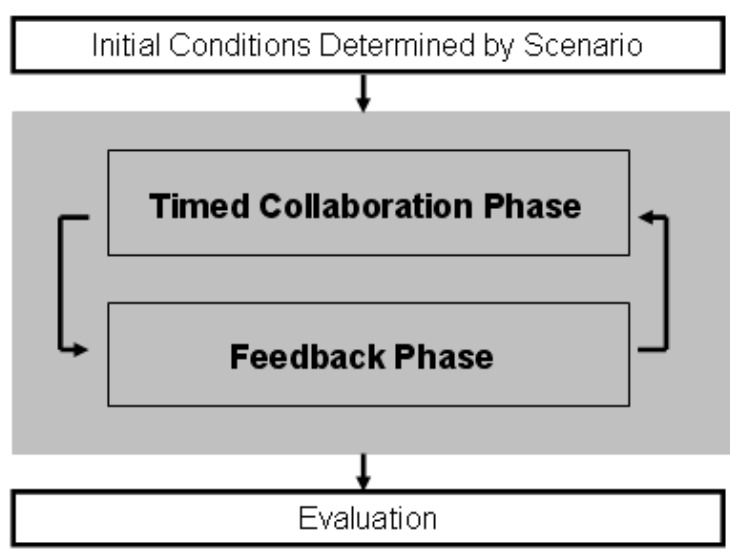

Fig. 1. Illustration of the structure of a game play session

The specificity of feedback provided by a manual or cognitive skills training program is a reliable predictor of future performance of those skills [10]. Specifically, feedback provided to participants by the game should be both immediate and specific to the actions taken. Driving instruction courses provide a good example of skills training methodologies. In these courses, the instructor examines the behavior of the learner as they drive and delivers timely and specific feedback concerning the proficiency of the learners' driving. Allowing a learner to drive for thirty minutes before producing a list of mistakes would not allow the learner to effectively discern which of the many actions taken were appropriate, and which were not.

It appears that an effective serious game designed to teach soft skills should involve game-based problemsolving that delivers clear, timely and specific feedback to players. The current project aims to fulfill these criteria in two ways. Firstly, it appears that the desired learning outcomes must be embedded within the game play mechanics. The learning outcome of the current project is improved group decision making skills. Hence, the game presents an environment where it is advantageous to engage in the appropriate group decision making and communication behaviors. Groups that do not work collaboratively to solve the problems presented in the game should perform poorly. Thus, the learning is embedded within the game play mechanics and the game state itself provides feedback on how well the group is performing in terms of soft skills.

Secondly, the game design breaks long and complex disaster scenarios into a number of shorter, simpler ones. Essentially, there are a number of short collaboration sessions where participants can collaborate to alleviate the immediate risks presented by the dynamically changing game state. These collaboration sessions are separated by short feedback phases, where a tutor has the opportunity to deliver specific feedback to groups or individuals with reference to the behavior observed in the previous collaboration phase, in order to improve the collaborative processes of the group.

A table top prototype of the soft skills training game is reported below. Table top prototyping is an established method of refining a game design concept into a fully functional game [11]. The process typically involves the creation of a board game that encapsulates the game mechanics desired for the final game. Participants play this board game while designers observe the balance of variables within the game, analyze the pattern of information flow between players and refine the game mechanics, where appropriate [12]. The following section describes the table top prototype created during the development of a soft skills training game.

\section{A BOARD GAME PROTOTYPE FOR SOFT SKILLS TRAINING}

The game reported here places players in a crisis management team that is dealing with an immediate and developing emergency situation. Figure 1 presents an illustration of the structure of a game play session. There are three stages to each session. Initially, conditions are set by the particular scenario chosen for the training session. Game play is organized into a series of timed rounds, each separated by a phase in which a tutor has the opportunity to give feedback to players. Once the prescribed number of timed rounds have elapsed, an indepth evaluation phase is initiated between the tutor and the participants.

It must be noted that for the purposes of the initial evaluation reported in this paper, the role of tutor was left unfulfilled. Rather, this trial was concerned with evaluating whether the game play mechanics did or did not require participants to engage in the sorts of soft skills that the DREAD-ED project aims to teach. As such, the feedback phase in this evaluation merely consisted of a short break from the collaborative sessions and an opportunity to organize the game pieces.

The game mechanic is based on assembling teams of similarly skilled personnel in order to affect the game state, as represented by a number of scales, thereby controlling the emergency situation. The personnel are represented by a deck of custom made cards, each one displaying the name of the personnel class to which that card belongs, plus a function that it has within the game. Players receive six of these cards each and have the option of exchanging cards with other players, or with a reserve of unseen cards. In addition, each player is randomly assigned a particular role that allows them to alter the game state in a very specific way. A player's role is to collaborate and negotiate with the other members of the team to manage the emergency situation by gathering together and deploying teams of specialists in order to reduce the impact of the developing emergency. A high-achieving group will excel at getting the right personnel to the right players at the right time in order to control the emergency. Figure 2 constitutes a graphic illustration of the scales used in the table top prototype to represent the game state.

A mechanism was developed to control the exchanging of cards between players, so that only a certain number of actions are possible per round. Specifically, each group was given a collective allocation of 'action pieces' that could be used in each collaboration phase. Groups had to decide collectively on how to use these action pieces. This mechanic, coupled with the limited time available for discussion and collaboration, was designed to engender the sort of strategic decision making typically necessary or managing emergencies. 
In addition, at specific points, 'injects' of information are introduced that alter the game state in an unpredictable fashion. These occur both at the beginning of each collaboration phase and when a player deploys a personnel team. In this way, the game presents an unpredictable dynamically changing environment that models the uncertainty and stress typically associated with managing emergencies.

It must be noted that the version of the game used for the current evaluation did not contain any narrative content beyond the names of personnel and role cards. Rather, a skeletal structure comprising just the values assigned to each card or class was included. This step was taken in order to concentrate purely on the effectiveness of the game mechanics, rather than the engagement created by a narrative structure. Future versions of the game will include detailed narrative structures as part of the game play scenarios.

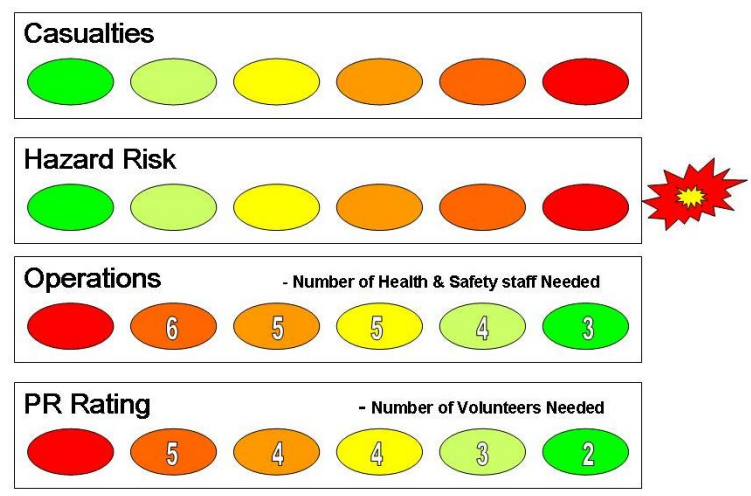

Fig. 2. An illustration of the scales used in the table top prototype to represent the game state.

Twenty-four undergraduate students were recruited for the study and assigned to four groups of six participants in an ad-hoc manner. No effort was made to carefully construct groups as representative samples of the population, as we were not interested in extrapolating generalized findings about the participants themselves, or group processes in general. This study was an initial evaluation of the game design only, and as such was conducted purely to evaluate whether the game design provoked collaborative behavior.

In order to evaluate the effectiveness of the board game as a tool for training soft skills, the four groups were casually observed by researchers while playing the game. As an aside, an objective method of quantifying collaborative behavior in games is being developed also as part of the DREAD-ED project, but this was not available at the time of the current study. Additionally, questionnaires that probed the game play mechanics, strategies developed by players and weighting issues were distributed on completion of game play.

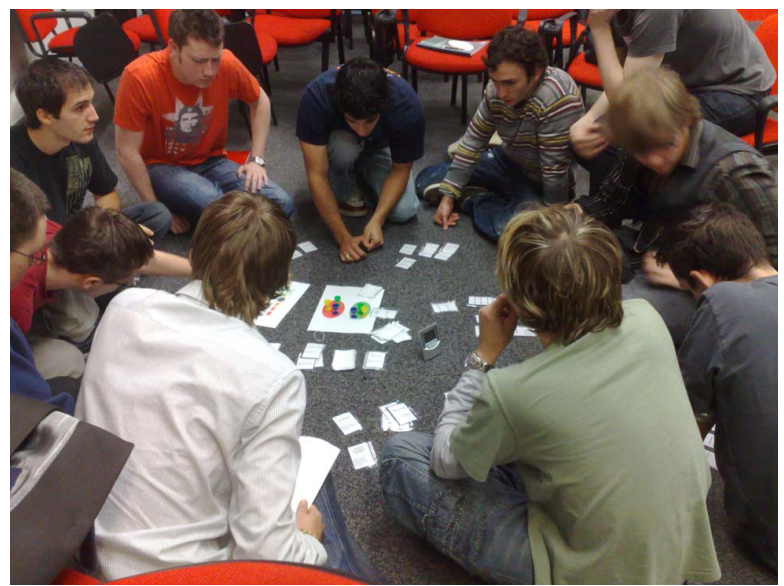

Fig. 3. Participants playing the table top prototype game.

Observations indicated that in three of the four sixmember groups, there appeared to be a great deal of communication between group members and forward planning was in evidence. These groups appeared to be highly engaged with the game and seemed to display very good collaborative decision-making. In addition, group participation appeared to become more equal as the game progressed. In essence, the game appeared to produce an environment in which it was advantageous to engage in the skills that were targeted at the outset of the project. The remaining group communicated less well throughout, and consequently performed poorly at the game. At the moment, the question of 'why' one group performed worse than the others is beyond the scope of this research. Rather, it is important to note that while this group did not seem to be adapting their behavior according to the feedback provided dynamically by the game state, in a full version of the game, the feedback provided by a tutor during the feedback phases may have been enough to provoke the appropriate collaborative behavior.

Questionnaire responses generally correlated with observations. Players reported that team work, communication and a lack of selfishness were essential for success at the game. One participant reported, "It was only once we began discussing our possible moves at the beginning of every turn that we managed to stabilize our situation in the game. The game forced us to work as a team, or fail." It appears that the design is fit for the purposes intended; creating an environment where it is advantageous to engage in positive and collaborative group decision making, while promoting communication skills.

\section{CONCLUSIONS AND FURTHER WORK}

Initial findings suggest that the game design adopted in the current project successfully created an environment where it was advantageous to engage in collaborative group decision making. Three of the four groups that participated displayed collaborative and inclusive decision making processes that were refined over successive rounds. Crucially, the game design, where there are a large number of feedback stages in between the timed game play phases, allows scope for a tutor to identify unproductive behaviors in individuals and groups, and to 
help engender more appropriate behaviors through rehearsal.

The board game reported in the current paper represented the skeletal structure of the game mechanic. Players were managing numbers and cards, rather than information relevant to emergency management. The next step in the design process is to create a board game that includes a narrative structure, but maintains the mechanics of the basic prototype.

As mentioned above, a methodology for quantifying collaborative behavior in games is also currently under development as part of the DREAD-ED project. This methodology will assess the structure and quality of group decision making processes within the game, based on both objective observations and social network analyses. In addition, it is intended that this methodology will be refined and validated over an extended period, in order to serve as a useful tool both for this project and other collaborative environments. Crucially, it is intended that this tool will plug into the final online version of the soft skills training game.

\section{ACKNOWLEDGMENT}

This work was carried out as part of the "Leonardo" project "DREAD-ED: Disaster Readiness through Education" funded by the EU Lifelong Learning Programme (see http://www.dread-ed.eu/ $<$ http://www.dread-ed.eu/>

\section{REFERENCES}

[1] K. M. Kowalski-Trakofler, and T. Scharf, "Judgment and decision making under stress: an overview for emergency managers," International Journal of Emergency Management, vol. 1, no. 3, pp. 278-289, 2003.

[2] J. P. Kincaid, J. Donovan, and B. Pettitt, "Simulation Techniques for training emergency response," International Journal of Emergency Management, vol. 1, no. 3, pp. 238-246, 2003.
[3] S. Jain, and C.R. McLean, "Integrated Simulation And Gaming Architecture For Incident Management Training," Proceedings of the 2005 Winter Simulation Conference, pp. 904-913, M. E. Kuhl, N. M. Steiger, F. B. Armstrong, and J. A. Joines, eds., 2005.

[4] R.L. Sanders, and G.S. Rhodes, "A Simulation Learning Approach to Training First Responders for Radiological Emergencies," Proceedings of the 2007 summer computer simulation conference, San Diego, California, article no. 28, 2007.

[5] M. Crichton, and R. Flin, "Training for Emergency Management: Tactical Decision Games," Journal of Hazardous Materials, vol. 88, pp. 255-266, 2001.

[6] S. Alper, D. Tjosvold, and K.S. Law, "Interdependence and Controversy in Group Decision Making: Antecedents to Effective Self-Managing Teams," Organizational Behavior and Human Decision Processes, vol. 74, no. 1, pp. 33-52, 1998.

[7] N.L. Kerr, and R.S. Tindale, "Group Performance and DecisionMaking," Annual Review of Psychology, vol. 55, pp. 623-55, 2004.

[8] F.L. Greitzer, O.A. Kuchar, and K. Huston, "Cognitive Science Implications for Enhancing Training Effectiveness in a Serious Gaming Context," ACM Journal of Edcational Resources in Computing, vol. 7, no. 3, Article 2, 2007.

[9] I. Bogost, Persuasive Games. Cambridge, MA: MIT Press, 2007.

[10] A.C. Catania, Learning (4th ed.). Englewoods Cliffs, NJ: Prentice Hall, 1998.

[11] T. Fullerton, C. Swain, and S. Hoffman, Game Design Workshop: Designing, Prototyping, and Playtesting Games, San Francisco, CA: CMP Books, 2004.

[12] J. Carroll, H. Mentis, G. Convertino, M. Rosson, C. Ganoe, H. Sinha, D. Zhao, "Prototyping Collaborative Geospatial Emergency Planning," Proceedings of ISCRAM2007, B. Van de Walle, P. Burghardt, and C. Nieuwenhuis, eds, 2007. 